\title{
Zur Frage der Existenz von Selen-Jodverbindungen.
}

\author{
Von \\ Ernst Beckmann und Otto Faust. \\ Mit 1 Figur im Text.
}

Während SchNeIDER ${ }^{1}$ u. a. glaubten durch Zusammenreiben und Erhitzen oder auch durch chemische Umsetzungen ein Selenmonojodid, $\mathrm{Se}_{2} \mathrm{~J}_{2}$, und Selentetrajodid, $\mathrm{SeJ}_{4}$, erhalten $\mathrm{zu}$ haben, wurden durch neuere Untersuchungen von Pelulini und Pedrina, OuIvari, sowie E. BeckManN solche Verbindungen nicht bestätigt. Während aber Schwefel in Jodlösung das normale groBe Molekül $S_{7}$ bis $S_{8}$ ergeben hat, findet bei Selen in Jodlösung eine Aufspaltung des großen Moleküls $\mathrm{Se}_{8}$ bis $\mathrm{Se}_{10}$ in kleine Moleküle $\mathrm{Se}_{1}$ bis $\mathrm{Se}_{2}$ statt. Ein spezifischer EinfluB des Jods auf Schwefel ist bisher nur insofern beobachtet worden, als es die Bildung der amorphen Modifikation begünstigten soll und wie es scheint auch bei Selen die Entstehung der in Schwefelkohlenstoff unlöslichen Modifikation erleichtert.

Wir haben im nachfolgenden neue Versuche angestellt, ob nicht doch die Bildung lockerer Verbindungen dem dissoziierenden EinfluB des Jods auf Selen zugrunde liegt. Der erneute Versuch, aus den Schmelz- und Erstarrungskurven von Selen-Jodlegierungen zu einer sicheren Entscheidung zu kommen, scheiterte ebenso wie früher ${ }^{2}$ an der Neigung des Selens und der Selen-Jodlegierungen zur Unterkühlung und an der geringen Kristallisationsgeschwindigkeit. Es wurde daher versucht mit Hilfe einer Reihe von anderen Eigenschaften Einblick in die Konstitution der Selen-Jodlegierungen zu erhalten.

a) Volumenänderung beim Schmelzen.

Wenn zwei Stoffe zusammengeschmolzen werden und dabei weder eine Verbindung bilden, noch beim Erstarren Mischkristalle ausscheiden, wenn sie also lediglich in einem eutektischen Kon-

1 Pogg. Ann. 129 (1866), 627.

2 Vgl. E. Beckmann und E. Grünthat, Z. anorg. Chem. 84 (1913). 
glomerat erstarren und auch im geschmolzenen Zustande lediglich ein Gemisch der beiden Komponenten sind, so ist anzunehmen, daB die beim Schmelzen des Gemisches eintretende Volumenänderung sich für die Legierungen nach der Mischungsregel aus den Volumenänderungen beim Schmelzen der reinen Komponenten werden berechnen lassen, wobei man die erhaltenen Resultate für die reinen

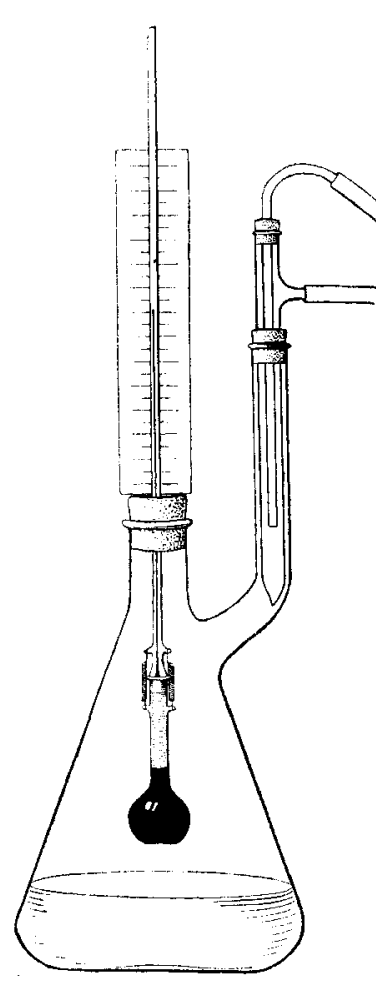

Fig. 1. Dilatometer.

(1/4 wirkl. Größe.)

Komponenten und die Legierung natürlich auf dieselbe Temperatur reduzieren muß.

Zur Ausführung der Versuche wurde eine bestimmte Menge der zu untersuchenden Substanz in ein als Dilatometergefä $B$ dienendes Kölbchen gebracht, an das mit Hilfe eines von der Firma Dr. Heinrich Göckel, Berlin, gelieferten vorzüglichen Schliffes ein Steigrohr angesetzt werden konnte (Fig. 1). Der Schliff wurde zur Vorsicht noch mit zwei kräftigen Federn zusammengepreßst und erwies sich bei den gemachten Versuchen als vollkommen dicht und brauchbar. Untersucht werden die Volumenänderungen beim Schmelzen für reines Jod, reines Selen und für die bei konstanter Temperatur schmelzende eutektische Legierung mit 50 Atomproz. Selen. Als Sperrflüssigkeit wurde für Selen mit gutem Erfolg reines Kahlbaumsches Paraffinöl verwendet. Für die eutektische Legierung konnte Wasser benutzt werden. Beide Stoffe waren jedoch für Jod nicht verwendbar. Paraffinöl löst Jod in großen Mengen und vor allem ist der Temperaturkoeffizient der Löslichkeit sehr erheblich, so daß eine bei Zimmertemperatur gesättigte Lösung von Jod in Paraffinöl beim Schmelzpunkt des Jods nicht annähernd gesättigt ist. Organische Substanzen lösen alle sehr leicht Jod. Ganz reine konzentrierte Schwefelsäure erwies sich jedoch als eine sehr gute Sperrflüssigkeit für Jod. Dieses löst sich nur sehr wenig darin auf und auch bei Temperaturen über $120^{\circ}$ trat noch keine merkbare Reaktion zwischen Jod und Schwefelsäure ein. Das Dilatometergefäß (Fig. 1) wurde mittels eines durchbohrten Korkes in einem Erlenmeyerkolben befestigt, der einen seitlichen Ansatz für Rückfluß. 
kühlung hatte. In dem Kolben konnte man Flüssigkeiten von bestimmtem Siedepunkt sieden lassen und so das Dilatometergefäß beliebig lange auf einer bestimmten Temperatur halten. Im allgemeinen stellte sich nach einigen Minuten die Flüssigkeit in der Steigröhre auf eine konstante Höhe ein, beim Schmelzen der Substanz mußte man etwas länger warten. Die Versuche sind mehrere Male wiederholt worden und zwar wurde bei jeder Versuchstemperatur der Stand beim Erhitzen und später wieder beim Abkühlen beobachtet. Diese beiden Ablesungen muBten übereinstimmen. Durch geradliniges Verlängern der Volumentemperaturkurven der festen und der geschmolzenen Substanz wurden die Volumenänderungen beim Schmelzen auf eine Temperatur von $170^{\circ}$ extrapoliert, d. h. eine ungefähr in der Mitte zwischen dem Schmelzpunkt rom Jod und vom Selen liegende Temperatur. Die so erhaltenen Resultate sind in der folgenden Tabelle angegeben. Die Volumenangaben beziehen sich auf die Skalenteile der willkürlichen Dilatometerskala:

Tabelle 1.

Volumenänderung beim Schmelzen von Selen-Jodlegierungen reduziert auf $170^{\circ} \mathrm{C}$.

\begin{tabular}{c|c|c|c}
\hline \hline $\begin{array}{c}\text { Konz. in } \\
\text { Atomproz. }\end{array}$ & bestimmt & $\begin{array}{c}\text { Volumen } \\
\text { nach Mischungsiegel } \\
\text { berechnet }\end{array}$ & Differenz \\
\hline \hline $\begin{array}{c}\text { 100 Jod } \\
\text { 50 Jod }+\end{array}$ & 4.15 & & \\
$\begin{array}{c}\text { 50 Selen } \\
100 \text { Selen }\end{array}$ & 2.62 & 2.83 & $0.21=7.4 \%$ \\
\hline
\end{tabular}

Die Genauigkeit der Bestimmung betrug $2-3 \%$ und da durch die Extrapolation leicht ein Fehler von einigen Prozenten gemacht werden kann, so ist zu folgern, daß innerhalb der Genauigkeitsgrenzen der Bestimmung die Volumenänderung beim Schmelzen der Selen-Jodlegierungen sich nach der Mischungsregel berechnen läßt, was darauf deutet, daB in Übereinstimmung mit den thermischen Resultaten von Peluini und Pedirina Selen und Jod miteinander wahrscheinlich keine Verbindung bilden, sondern einfach eutektisch erstarren.

b) Spezifisches Volumen der Selen-Jodlegierungen.

Weiterhin wurde das spezifische Volumen der Selen-Jodlegierungen untersucht. Hierbei war die analoge Überlegung maßgebend, wie bei der Bestimmung der Volumenänderung beim Schmelzen. 
Wenn die Selen-Jodlegierungen einfach als eutektisches Konglomerat erstarren, so ist sehr wahrscheinlich, daß das spezifische Volumen einer Legierung sich nach der Mischungsregel aus dem spezifischen Volumen der Komponenten berechnen läßt. Für Jod-Schwefellegierungen, welche nach allgemeiner Annahme keine Verbindung oder Mischkristalle bilden, sondern einfach eutektisch erstarren, ist dies ron OLIVARI ${ }^{1}$ untersucht und entsprechend gefunden worden.

Die Bestimmung des spezifischen Volumens geschah in der Weise, daß eine bestimmte Menge der zu untersuchenden Substanz in ein geeichtes Kölbchen gebracht und alsdann mit dem Kölbchen wieder gewogen wurde. Nach Einfüllen einer indifferenten Flüssigkeit von bekanntem spezifischen Gewicht bis zur Volumenmarke unter Entfernuug aller Luftblasen durch Erwärmen und Evakuieren mit einer LEYBoLDschen Ölpumpe wurde das gefüllte Kölbchen von neuem gewogen. Erhaltene Resultate sind in Tabelle 2 wiedergegeben.

Tabelle 2.

Spezifische Volumina der Selen-Jodlegierungen bei $19^{\circ}$.

\begin{tabular}{c|c|c|c}
\hline \hline Volumproz. & bestimmt & $\begin{array}{c}\text { Spez. Volumen } \\
\text { nach der Mischungs- } \\
\text { regel berechnet }\end{array}$ & Differenz \\
\hline \hline 100 Jod & 0.2034 & & $0.0001=$ \\
42 Jod & 0.2099 & 0.2098 & $0.05 \%$
\end{tabular}

Das spezifische Volumen der Selen-Jodlegierungen stimmt also innerhalb der Fehlergrenzen mit dem nach der Mischungsregel berechneten überein, es verhält sich genau so wie das spezifische Volumen der Jod-Schwefellegierungen, die unter Bildung eines einfachen Eutektikums erstarren. Das beruht darauf, daB die Selen-Jodlegierungen ebenfalls ein einfaches Eutektikum liefern.

Zur Prüfung dieser SchluBfolgerung auf ihre Berechtigung wurden auch die spezifischen Volumina der Tellur-Jodlegierungen untersucht. Tellur bildet mit Jod eine Verbindung von der Formel $\mathrm{TeJ}_{2}$. Die Resultate finden sich in der folgenden Tabelle 3.

Die Volumenausdehnung bei Bildung der untersuchten Legierung überschreitet die Genauigkeit der Bestimmung etwa um das Zehnfache. Hier also, wo sich bestimmt eine Verbindung bildet, ist das spezifische Volumen nicht mehr nach der Mischungsregel berechenbar.

${ }^{1}$ Rend. Ace. Lincei 17 II, Ser. 5 (1908), 513. 
Tabelle 3.

Spez. Volumen der Tellur-Jodlegierungen bei $19^{\circ}$.

\begin{tabular}{|c|c|c|c|}
\hline Volumproz. & bestimmt & $\begin{array}{l}\text { Volumen } \\
\text { nach der Mischungs- } \\
\text { regel berechnet }\end{array}$ & Differenz \\
\hline $\left.\begin{array}{rl}100 & \text { Jod } \\
28 & \text { Tellur } \\
72 & \text { Jod } \\
100 & \text { Tellur }\end{array}\right\}$ & $\begin{array}{l}0.2034 \\
0.1933 \\
0.1592\end{array}$ & 0.1905 & $\begin{array}{c}0.0028= \\
1.5 \%\end{array}$ \\
\hline
\end{tabular}

c) Das elektrische Leitvermögen.

Das elektrische Leitvermögen von Jod ${ }^{1}$ und besonders von Selen ${ }^{2}$ ist schon häufig untersucht worden. Das spezifische Leitvermögen von flüssigem Jod haben Lewrs und WHEeLer ${ }^{1}$ bestimmt. Nach diesen Autoren beträgt es zwischen 120 und $160^{\circ}$ etwa $0.6 \times 10^{-4}$ reziproke $\mathrm{Ohm}$. Ganz geringe Zusätze von Salzen erhöhen das Leitvermögen stark. Bei den hier beschriebenen Versuchen wurde das Leitvermögen in einem gewöhnlichen KoHLRAuschschen Widerstandsgefäß mit blanken Platinelektroden unter Anwendung von Wechselstrom eines kleinen Induktoriums mit WEHNELTUnterbrecher geprüft. Der Widerstand des mit $1 / 100^{-}$-norm. Kaliumchloridlösung gefüllten Gefäßes betrug bei $20^{\circ}$ etwa $300 \mathrm{Ohm}$. Die benutzten ziemlich gleichen GefäBe wurden vor den eigentlichen Versuchen stets mit $1 / 100$-norm. Kaliumchloridlösung geeicht.

Das spezifische Leitvermögen des untersuchten $\mathrm{K}_{\text {AmLBADM schen }}$ Jods betrug bei $112^{\circ}$ im flüssigen Zustand $0.695 \times 10^{-3}$, also das Zehnfache des von Lewis und Wheeler gefundenen Wertes. Dabei ist zu berücksichtigen, daB schon einige Hundertstel Prozent eines Salzzusatzes diese Erhöhung des Leitvermögens herrorrufen können. Diese Mengen vermag sich das Jod wohl aus dem Glase des Widerstandsgefäßes zu lösen. Auch war das Platin der Elektroden vom Jod etwas angegriffen und mag zur Vergrößerung der Leitfähigkeit mit beigetragen haben. Bein Erstarren sank das Leitvermögen außerordentlich rapid, betrug bei $111^{0} 0.16 \times 10^{-7}$ und war bei Zimmertemperatur unmeBbar klein.

Geschmolzenes Selen leitete den elektrischen Strom gar nicht. Beim Abkühlen erstarrt die Schmelze zu einer amorphen Masse,

\footnotetext{
1 Zeitschr. phys. Chem. 56 (1906), 183.

2 Vgl. Gmelin-Kratt, Handbuch, Bd. I, Abt. 1 (1907), 732.
} 
die ebenfalls absolut nichtleitend ist. Erhitzt man diese Masse nur einige Minuten auf etwa $170-180^{\circ}$, so wird sie leitend, zunächst wenig; die Leitfähigkeit nimmt jedoch mit der Erhitzungsdauer zu, nach 16 stündigem Erhitzen auf $197^{\circ}$ betrug das spezifische Leitvermögen bei dieser Temperatur $0.592 \times 10^{-2}$, also etwa die Hälfte des spez. Leitvermögens der $1 / 100^{-n o r m}$. Kaliumchloridlösung bei $20^{\circ}$. Der Temperaturkoeffizient des Leitvermögens dieser Selenmodifikation hat metallischen Charakter, das Leitvermögen steigt also mit sinkender Temperatur; es betrug bei $131^{\circ} 0.902 \times 10^{-2}$, bei $110.6^{0} 0.961 \times 10^{-2}$. Unterhalb etwa $80^{\circ}$ nimmt das Leitvermögen mit sinkender Temperatur $a b$ und betrug $z$. B. bei der Untersuchung der für obige Versuche benutzten Probe bei $21.4^{0} 0.44 \times 10^{-2}$. Wurde diese Probe wieder langsam im Verlauf von 5 Stunden auf $200^{\circ}$ erhitzt, so stieg jetzt, bis zu einer Temperatur von $177.5^{\circ}$, das Leitvermögen auf $0.66 \times 10^{-2}$, oberhalb dieser Temperatur sank das Leitvermögen wieder. Bei $197.6^{\circ}$ betrug das Leitrermögen $0.578 \times 10^{-2}$, also annähernd das gleiche, wie vorher bei derselben Temperatur. Ganz reversible Werte des Leitvermögens waren für Selen nicht zu erhalten, auch nicht, wenn man die Vorsicht gebrauchte, ganz im Dunkeln zu arbeiten. Ein Einfluß des Lichtes war überhaupt bei diesen Versuchen nicht zu konstatieren. Sowie man das bis auf $200^{\circ}$ erhitzte Selen weiter bis zum Schmelzen erhitzte, sank das Leitvermögen rapid und betrug wieder Null, wenn alles Selen geschmolzen war.

Von den Selen-Jodlegierungen wurde wieder das eutektische Gemisch von 50 Atomproz. Jod und Selen untersucht. Das geschmolzene Gemisch hatte bei $58^{\circ}$ ein spez. Leitvermögen von $0.382 \times 10^{-3}$, also von derselben GröBenordnung wie früher das geschmolzene Jod, dessen Leitvermögen zu $0.695 \times 10^{-3}$, also ungefähr doppelt so groß, gefunden war. Beim Abküblen nahm die Leitfähigkeit der unterkühlten Schmelze ab und betrug bei $2^{\circ}$ $0.145 \times 10^{-3}$, bei $20^{0}$ begann die Schmelze zu erstarren und die Leitfähigkeit nahm dabei sehr stark ab. Das Erstarren der Legierung geht sehr langsam vor sich. Nach einigen Stunden war eine Leitfähigkeit überhaupt nicht mehr festzustellen.

Die Legierung zeigte also in bezug auf das Leitvermögen qualitativ die gleichen Eigenschaften, wie das vorher erstarrte reine Jod. Das Selen, das geschmolzen und amorph erstarrt im reinen Zustande zunächst keine Leitfähigkeit besitzt, scheint auch in der Jod-Selenlegierung einen Beitrag zur Leitfähigkeit nicht zu liefern. 
Die Legierung enthält 58 Volumproz. Jod und dementsprechend ist das Leitrermögen verkleinert, es beträgt nur $63.3 \%$ des bei reinem geschmolzenen Jod gemessenen Leitvermögens. Es hat hiernach durchaus den Anschein, daB die eutektische Selen-Jodlegierung sich wie ein mechanisches Gemisch von Selen und Jod verhält, und zwar scheint es, daB beim Erstarren nur das Jod zunächst kristallinisch wird, während das Selen seine Eigenschaft, sich auBerordentlich leicht unterkühlen zu lassen, auch in der Legierung beibehält und auch hierbeim FestwerdenderLegierung zunächst amorph erstarrt. Hierfür spricht auch die folgende Erscheinung: Wenn man die erstarrte Selen-Jodlegierung mehrere Stunden bei einer Temperatur von $46^{\circ}$ hält, so zeigt sie allmählich ein Leitvermögen, das nach 12 stündiger Versuchsdauer zu $1.076 \times 10^{-3}$ bestimmt wurde und nach 72 stündigem Erbitzen sogar, bei $46^{\circ}$, zu $3.0 \times 10^{-3}$. Bei weiterem Erhitzen nahm das Leitvermögen immer noch sehr langsam zu. Die feste Legierung zeigte jetzt also in bezug auf das Leitvermögen die Eigenschaften, die amorph erstarrtes Selen nach dem Erhitzen zeigt. Auch die Größenordnung des Leitvermögens der Legierung ist die gleiche wie bei reinem Selen, dessen Leitvermögen nach dem Erstarren und Wiedererhitzen bei $21.4^{0}$ zu $4.4 \times 10^{-3}$ bestimmt wurde. Auch der Temperaturkoeffizient der Legierung hatte, was wichtig ist, dasselbe Vorzeichen wie beim reinen Selen, wo er bei etwa $80^{\circ}$ und darunter positiv ist. Beim Abkühlen auf $23.3^{\circ}$ wurde das Leitvermögen der Legierung zu $0.91 \times 10^{-3}$ bestimmt. Also auch in diesem Falle verhält sich die Jod-Selenlegierung wie ein Gemisch aus Jod und Selen und das nach dem Wiedererwärmen erhaltene Leitvermögen der festen Legierung ist nunmehr auf Kosten des Selens zu setzen, das ebenfalls, amorph erstarrt, kein Leitvermögen besitzt und erst nach darauffolgendem Erhitzen ein solches aufweist. Festes Jod zeigt auch nach abermaligem Erhitzen kein Leitvermögen. Auch die Größe der für das Leitvermögen erhaltenen Zahlen entspricht den nach dieser Auffassung zu erwartenden Werten recht gut.

Es wurde weiter das Leitvermögen von Schwefel-Jod- und ron Tellur-Jodlegierungen untersucht, um festzustellen, ob diese Legierungen in ihrem Leitvermögen ein Verhalten zeigen, das dem thermischen Zustandsdiagramme entspricht.

Das eutektische Gemisch ron Jod und Schwefel zeigte im geschmolzenen Zustand eine Leitfähigkeit von etwa $0.1 \times 10^{-2}$, die erstarrte Masse besa $B$ jedoch nicht mehr das geringste Leitvermögen. 
Reiner Schwefel hat so gut wie kein Leitvermögen. ${ }^{1}$ Also auch diese Legierung verhält sich wie ein Gemisch von Jod und Schwefel, wie nach ihrem thermischen Verhalten zu erwarten war.

Ein etwas anderes Verhalten zeigen die Jod-Tellurlegierungen. Untersucht wurde die Verbindung $\mathrm{TeJ}_{2}$. Diese hat im geschmolzenen Zustande bei $181^{\circ}$ ein Leitrermögen von $138 \times 10^{-2}$. Dasselbe ist also immerhin erheblich größer als die bisher gemessenen Leitfähigkeiten. Quecksilber hat bei $0^{0} 1.06 \times 10^{+4}$. Reines Tellur besitzt bei $19.6^{\circ}$ ein Leitvermögen von $4.66 \times 10^{+4}$, es ist also erheblich gröBer als bei $\mathrm{TeJ}_{2}$. Das Leitvermögen der geschmolzenen Legierung nimmt mit sinkender Temperatur $a b$, beträgt bei $163^{\circ} 19.3 \times 10^{-2}$ und beim Schmelzpunkt zeigt sich ein Sprung; die Leitfähigkeit nimmt sehr stark ab, beträgt bei $144^{\circ}$ noch $0.207 \times 10^{-3}$, bei $125^{0}$ noch $0.099 \times 10^{-3}$ und ist schon bei $40^{\circ}$ unmeBbar klein. Während das reine Tellur ein metallisches Leitvermögen mit negativem Temperaturkoeffizienten hat, zeigt die Leitfähigkeit der Verbindung $\mathrm{TeJ}_{2}$ einen metalloiden Charakter und verbält sich durchaus anders als ein mechanisches Gemisch von Jod und Tellur, wie auch aus dem thermischen Verhalten zu erwarten war, nach welchem sich beide Komponenten zu einer Verbindung $\mathrm{TeJ}_{2}$ vereinigen. Die Jod-Schwefel- und Jod-Selenlegierungen jedoch, die, nach ihrem thermischen Verhalten zu schließen, keine Verbindung, auch keine Mischkristalle, miteinander bilden, verhalten sich auch in bezug auf ihr elektrisches Leitvermögen wie ein einfaches mechanisches Gemisch der beiden Komponenten.

d) Kryoskopische Bestimmungen in Gegenwart von Lösungsmitteln.

Um auf einen dissoziierenden EinfluB des Jods gegenüber Selen zu prüfen, schien uns die kryoskopische Bestimmung dieser beiden Elemente nebeneinander in einem indifferenten Lösungsmittel wünschenswert. Als solches bot sich Methylenjodid dar, in welchem Selen wie auch Jod bereits früher von E. BeCKmanN ${ }^{2}$ gleich $\mathrm{Se}_{10}$ bzw. $J_{2}$ gefunden worden ist.

(Siehe Tabelle, S. 111.)

Aus diesen Versuchen geht hervor, daß bei der Schmelztemperatur des Methylenjodids $+4^{0}$, das Jod weder vom Selen gebunden noch das große Selenmolekül weitgehend gespalten wird. DaB früher

1 Vgl. Gmein-KraUt, Handbuch, Bd. 1, Abt. 1 (1907), S. 362.

ZZeitsehr. phys. Chem. 46 (1903), 853. 
Bestimmungen ron Selen und Jod im Methylenjodid.

Niedriger Schmelzpunkt $=+4^{0} ; 1 \quad K=137$.

\begin{tabular}{c|c|c|c}
\hline \hline $\begin{array}{c}\text { Methylenjodid } \\
g\end{array}$ & $\begin{array}{c}\text { g Substanz } \\
\text { (Einzelmengen addiert) }\end{array}$ & Erniedrigung & $\begin{array}{c}\text { Molekular- } \\
\text { gewicht }\end{array}$ \\
\hline \hline
\end{tabular}

49.5

49.5

1. Molekulargewicht von rotem gefällten Selen

2. Molekulargewicht von Jod in der Methylenjodid-Selenlösung

49.5

0.165 \begin{tabular}{l|l}
0.020 & $719=$ Se 9.1 \\
0.041 & $658=$ Se 8.3
\end{tabular}

$264=J 2.08$

in Methylenjodid das Molekulargewicht des Selens $=\mathrm{Se}_{\mathbf{1 0}}$, hier aber etwas niedriger gefunden wurde, ist nur auf Versuchsfehler wegen der geringen Konzentration zurückzuführen. Wieweit die Gegenwart des Methylenjodids das Jod in seiner Wirkung beeinfluBt, muB dahingestellt bleiben. Schon jetzt möchten wir darauf hinweisen, $d a B$ nach neuen Versuchen auch bei Gegenwart von Schwefel, und zwar bei der "natürlichen" Erstarruugstemperatur desselben $\left(114.5^{\circ}\right)$, welche mit der des Jods (114 ${ }^{\circ}$ ) nahezu übereinstimmt, eine Einwirkung von Jod auf Selen nachweisbar nicht stattfindet. Allerdings ist hier nicht bekannt, mit welchem Molekulargewicht das Selen in Schwefel sich löst.

Nachdem sich immer wieder gezeigt hat, wie wenig Jod geneigt ist, mit Selen eine Verbindung einzugehen, bleibt nur die Annahme übrig, daß ein anderer dissoziierender Einfluß des Jods bei der Wirkung auf dasselbe, ohne Dazwischentreten weiterer Lösungsmittel, zu den kleinen Selenmolekülen geführt hat. Dabei ist daran zu erinnern, ${ }^{2} \mathrm{da} b$ das Jod eine kleine Dielektrizitätskonstante, $4.0^{3}$ (gegenüber 80 bei Wasser) besitzt und nach OLIVARI organische Substanzen mit Hydroxylgruppen die Neigung zur Assoziation verraten. Außer elektrolytischer und thermischer Dissoziation dürften noch andere unbekannte Einflüsse nichtwässeriger Lösungsmittel sich geltend machen können. Dieselben waren auch in Betracht zu ziehen in den Fällen, wo die Molekulargewichte bei Dampfdichtebestimmungen in hoher Temperatur gröBer gefunden werden als innerhalb an-

${ }^{1}$ Der höhere Schmelzpunkt liegt bei $4.47^{\circ}$ und nicht bei $4.7^{\circ}$ wie in der zitierten Abhandlung mehrfach fehlerhaft gedruckt ist.

2 E. BeckManN, $Z$. anorg. Chem. 80 (1913), 222.

${ }^{3}$ In meiner früberen Abhandlung: $Z$. anorg. Chem 80 (1913), 234, ist der zu hohe Wert 10 von Ouivari, Rend. Acc. Lineei 18 II, Ser. 5 (1909), 384, übernommen worden; der Wert 4.0 ist von W. Sснмпрт bestimmt, Ann. d. Phys. [4] $11(1903), 120$. 
112 E. Beckmann und O. Faust. Zur Frage der Existenz usw.

scheinend indifferenter Lösungsmittel bei weit niedrigerer Temperatur. So gibt Aluminiumchlorid noch bei $218-432.7^{\circ}$ im Dampfzustande Moleküle mit Doppelatomen, während ätherische Lösungen schon bei $35^{\circ}$ Moleküle mit Einzelatomen geben. ${ }^{1}$ Auch Kupferchlorür enthält in Dampfform noch bei $1691^{\circ}$ größere Moleküle als innerhalb Chinolin bei $233^{\circ}{ }^{2}$ Man könnte dabei an eine Art Vergesellschaftung oder Anpassen des Lösungsmittels denken, welche zu einer Durchdringung der gelösten Substanz mit Molekülen des Lösungsmittels führt, ohne daß es zu einer Verbindung im gewöhnlichen Sinne kommt. Kürzlich haben W. A. Plotnikow und W. E. Rokot$J_{A N}{ }^{3}$ in ähnlicher Weise das elektrische Leitvermögen von JodBromlösungen auf elektrochemisches Anpassen der gelösten Substanz an das Lösungsmittel zurückgeführt, da Brom bei seiner kleinen Dielektrizitätskonstante ebenfalls nicht $\mathrm{zu}$ den ionisierenden Lösungsmitteln zu rechnen ist.

l E. Beckmann, Zeitschr. phys. Chem. 46 (1903), 857.

${ }^{2}$ E. Beckmanr, Z. anorg. Chem. 51 (1906), 240.

s Zeitschr. phys. Chem. 84 (1913), 365.

Berlin-Dahlem, Kaiser Wilhelm-Institut für Chemie, 12. September 1913.

Bei der Redaktion eingegangen am 15. September 1913. 